\title{
Supporting Multiple-tier QoS in a Video Bridging Application
}

\author{
Rajeev Koodli and C. M. Krishna \\ Department of Electrical and Computer Engineering \\ University of Massachusetts \\ Amherst, MA 01003, U.S.A. \\ phone: 413-545-0715, fax: 413-545-1993 \\ e-mail: koodli,krishna@ecs.umass.edu
}

\begin{abstract}
With the emergence of multimedia technology, it has become important to be able to support a diverse set of client applications (simply called applications henceforth), each with its own Quality-of-Service (QoS) requirement. Such soft real-time applications exhibit remarkable loss tolerance capacity, provided the losses are well regulated. In this paper, we propose the notion of a noticeable loss, which directly relates loss pattern to the perceived QoS for an application, and use it to evaluate a novel resource management algorithm that attempts to provide acceptable QoS to individual applications. We apply our model to a real-world application, and provide simulation results to illustrate the performance of this algorithm.
\end{abstract}

Keywords: deadlines, loss, end-to-end, scheduling

\section{Introduction}

With the dramatic improvements in processor and network speeds and data compression techniques that have occurred over the past few years, it has now become possible to support highly demanding multimedia applications such as video teleconferencing. However, such applications each have a certain Quality of Service (QoS) requirement, which translates to delay-sensitive demand on the underlying resources. In order to exploit the gains in hardware and support multiple multimedia applications with their respective QoS requirements, application scheduling becomes critical. This paper addresses the issue of scheduling applications so that their respective QoS requirements are satisfied in a Video Bridging system that facilitates live video conferencing. 


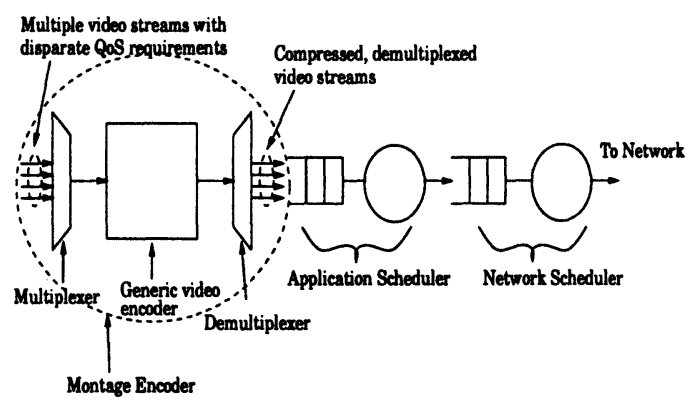

Figure 1: A Video Bridging Server Architecture

It is important to note that an application such as video conferencing requires access to multiple resources such as an encoder, the operating system (for access to memory and $\mathrm{I} / \mathrm{O}$ ) and the network. Hence, it becomes important to meet its QoS requirements at each of the individual resources which are shared by other applications, even though the application itself does not specify how its end-to-end QoS requirements have to be met at the individual resource nodes. Thus, effectively the scheduling problem becomes one of representing the QoS at each node and supporting it locally for a variety of applications.

The QoS provided to any application is a function of the scheduling at (a) the network, and (b) the end-system server such as a video bridge. Ideally, there would be one scheduler managing resources at both the network and the server. Such a scheduler would have perfect knowledge of the current state of the entire system (network plus server), and would therefore be able to manage resources efficiently. However, such a global scheduler is infeasible, and we must make do with a distributed scheduling solution. The overall management strategy is to divide the problem into network and server components, and to solve them separately. That is, we decompose the overall scheduling problem into separate network and server scheduling problems. Even at the server, it is frequently infeasible to have a scheduler overseeing all the scheduling there: quite often, the server itself consists of many subunits, for which a distributed scheduler is the best practical solution. While much work has been done in supporting QoS requirements in the networks area $[1,3,8,9]$, relatively little research has been done to provide desired QoS levels in end-system servers. That is the topic of this paper.

In this paper, we use a performance metric called Noticeable Loss Rate for evaluating the QoS offered to an application by a server. A task belonging to an application is considered lost if it misses its end-to-end deadline. The loss of a task is said to be noticeable if it occurs within an interval bounded by its previous loss instance and an application-specified time bound $\delta$. Using NLR as a performance metric, we introduce the approach of Preemptive Abortion and Cycle Stealing (PACS), which attempts to reduce the NLR of various applications by exploiting their respective time bounds, $\delta$, which we call loss constraints. PACS carries out scheduling at individual resource nodes such that all applications are provided an acceptable NLR. The effectiveness of the PACS scheme is evaluated using simulation experiments.

As a motivation for this work, consider the video bridging system shown in Fig- 
ure 1. This set-up is borrowed from a prototype at Bell Laboratories[4]. The input to the Multiplexer can arrive from different applications with disparate QoS requirements, i.e., the streams can possess different rates of frame generation, deadlines and loss tolerance. Such a sharing of a bridging system among different applications is often necessary for financial reasons. Once we have multiple applications contending for the same resource, scheduling becomes critical. The demultiplexed streams belonging to different applications will have to be scheduled by an Application Scheduler based on a delay-sensitive scheduling discipline such as Earliest Deadline First, or other real-time scheduling disciplines such as Rate Monotonic [10] or Cyclic Executive [2] in order to meet their timing requirements. So, the queue associated with the Application Scheduler in Figure 1 can be a priority-ordered queue based on deadlines (or some other parameter) or a simple FIFO queue that is serviced cyclically. The output of the Application Scheduler is serviced by the Network Scheduler which breaks the application data into smaller sized cells and transmits them to the network. Note that the Network Scheduler may augment scheduling with traffic compliance policies, as in ATM systems. The video streams have to traverse many resources such as the Encoder, the Application Scheduler (typically the OS) and the Network Interface Unit. In summary, it is clear that a facility such as a video bridging system will have to support multiple applications with individual QoS requirements and such support will have to be on an end-to-end basis.

The rest of the paper is organized as follows. We describe the system model and provide an overview of the solution approach in Section 2. The algorithm is described in Section 3, and numerical results are provided in Section 4. We conclude with a brief discussion in Section 5 . Due to space limitations, the literature survey and parts of the original paper have been moved to [7].

\section{System Model}

We make the following assumptions in this paper.

1. There are multiple applications contending for the available resources. Applications are classified according to their arrival pattern and QoS requirement. There are multiple classes of applications, each with its own distinct arrival pattern and QoS requirement. Each application class has its own priority assigned to it by a higher level entity such as a Call Admission Process, which is orthogonal to the scheduling problem addressed in here.

2. An application with end-to-end processing requirement generates complex tasks. Each complex task consists of various subtasks which execute at different resources. For example, processing a video frame generated by a camera is a complex task, consisting of subtasks for processing at the Encoder, the Operating System and the Network Interface. Subtasks of complex tasks belonging to different applications compete for resources.

3. An application that generates complex tasks specifies an end-to-end deadline and an acceptable noticeable loss rate. The end-to-end deadline is the time by which the processing has to be completed at the end-system server such as the video bridge shown in Figure 1. The subtasks of a complex task have no deadlines associated with them; the only specified deadline is the end-to-end deadline associated with the complex task. A complex task which misses its end-to-end deadline is assumed 


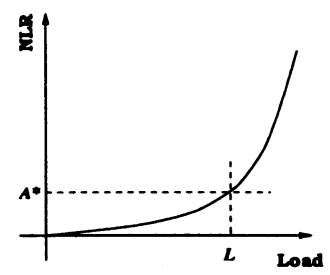

Figure 2: QoS Function

lost.

The loss of a complex task of an application belonging to a class $i$ is said to be noticeable if the interval between its loss and the previous loss for that application is no greater than $\delta_{i}$, where $\delta_{i}$ is a loss constraint specified for that application class. QoS is specified in terms of noticeable loss rate, NLR.

Example 1. Figure 2 shows the QoS function for some application class. $A^{*}$ is the maximum acceptable NLR for that application class, i.e., if the noticeable loss rate is greater than $A^{*}$, the QoS is no longer acceptable. Ideally, a scheduling algorithm should ensure that all applications are provided acceptable QoS. Note that the loss constraint and the acceptable NLR (ANLR) are related. For example, if $\delta$ is 1 second, then, in the worst case, a task may be lost every 1 second and the QoS will still be acceptable to the application. Within a window of 1 second, if 30 tasks are generated, then the ANLR is $1 / 30$, i.e., 3.33\%.

The concept of a noticeable loss is important to effectively capture the loss tolerance capacity of soft real-time multimedia applications. It imposes a restriction on how a service provider such as a network or a server may carry out resource management while supporting a large number of requests and during periods of overload. Typically, most resource management strategies simply abort tasks during overloads; some actually do so within the bounds of normally accepted aggregate task loss. For example, an application may specify an aggregate task loss of $3 \%$, but, this specification may allow too many consecutive losses to occur within a short window of time, which results in poor perceived quality for an application. Consecutive losses result in poor QoS for an application [13]. By placing a time bound on successive task losses, the concept of a noticeable loss forces a service provider to adhere to providing better perceived quality for an application.

4. We do not know the exact execution time of each subtask; however, we do have an estimate.

Given these conditions, we now address the problem of providing acceptable NLR for all applications belonging to various classes.

\section{The Algorithm}

There are two parts to our approach. In the first part, we concentrate on assigning virtual deadlines to subtasks so that their timely processing improves the chances of meeting the end-to-end deadline of the complex task that they are made of. 
In the second part, we present a resource management scheme that attempts to meet the QoS requirements of various applications during periods of overload and unpredictable service requirements. The algorithm works with a given call admission control (CAC) process. Its impact on the CAC, though beneficial, is not the subject of this paper.

\subsection{Assigning Nodal QoS parameters}

An application only specifies an end-to-end deadline and an end-to-end NLR. These values have to be apportioned among various resources to facilitate nodal (i.e., corresponding to individual resource nodes) support including call admission and scheduling. Assigning nodal deadlines, which we call virtual deadlines, follows a similar approach to other work [5], but differs from them in how it assigns virtual deadlines. The subtasks belonging to various complex tasks are assigned virtual deadlines based on their flexibility, which is defined as the ratio of available slack to estimated service time. The virtual deadlines are derived as follows.

$$
d_{t}=s_{x}+s_{x} /\left(\mu d_{f}\right)
$$

In the above expression, $\mu$ is the mean task service time, and $s_{x}$ is the estimated service time. $d_{f}$ is used as a control variable to vary the second term on the RHS, which represents the amount of estimated available slack to a subtask. If we take the ratio of the second term on the RHS to the first term in Equation (1), we get $1 /\left(\mu . d_{f}\right)$, which is a constant for all the subtasks. Thus, the above assignment allocates equal flexibility to all subtasks at a node. Experimental work has shown equal flexibility to be a superior approach [5] (a detailed description of the aforementioned assignment, including its performance is presented in [6]).

Recall that each application is associated with a loss constraint. This constraint can be apportioned among the various resource nodes of the server, thus converting a single loss constraint to individual loss constraints for each of these nodes. The apportionment process can follow any heuristic; in our own runs, we divide the loss constraint equally among all the nodes [9].

\subsection{Handling Overloads and Unpredictable Service Times}

The flexibility-based deadline assignment results in a fair share of the resource for all subtasks and hence enhances their chances of meeting the deadline requirement. However, as the load increases (i.e., as more requests are admitted), subtasks may miss their assigned deadlines due to the variance in processing times. It is important to note that a virtual deadline expiration need not result in subtask abortion. Instead, an attempt may be made to extract more processing time for such subtasks. It is clear that any scheme that attempts to extract more time for a tardy subtask would have to respect the QoS requirements of other tasks.

We begin presenting the Preemptive Abortion and Cycle Stealing (PACS) scheme with a simple algorithm that follows a dual-timeline verification. See Figure 3. The estimated processing times of the two subtasks are shown by the rectangular boxes. In step 1, the algorithm verifies if a subtask in the task queue has already missed its assigned deadline. If it has, it is simply skipped and the verification is done for the 


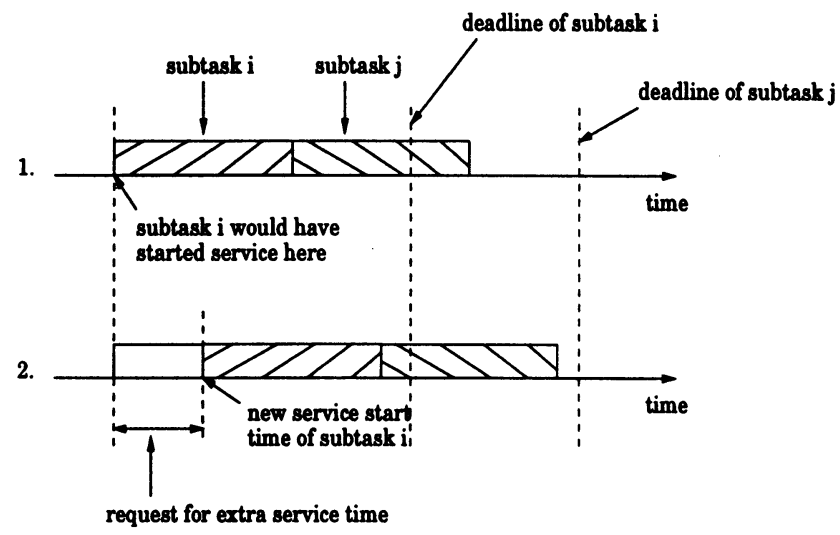

Figure 3: Dual-timeline verification

next subtask in the queue. If a subtask has not already missed its assigned deadline, then, in step 2, the algorithm verifies if it will still meet its assigned deadline if the requesting subtask is allowed to continue in service. If so, the process is repeated for the next subtask in the queue. If no subtask that has not already missed its assigned deadline will miss it as a result of extending service to the requesting subtask, the request is honored. We now extend the concept of dual-timeline verification to allow for preemptive abortion. Note that in step 2 of the dual-timeline algorithm, a bottleneck subtask (one whose assigned deadline would expire if the requesting subtask is allowed to continue) may be eligible for preemptive abortion. A subtask is said to be eligible for preemptive abortion if the previous instance of loss associated with its application and the current time differ by more than the application's nodal value of the loss constraint, since, then we can abort such a subtask without affecting the NLR for the application. Such an abortion can yield valuable time for a tardy subtask which might be in danger of experiencing a noticeable loss.

The PACS algorithm is invoked when an assigned deadline expires without the subtask having finished its execution. At this time, the system scheduler - which manages the scheduling of various subtasks at a resource - has to decide whether it should allow the subtask to continue in service for an additional time, which is again estimated. The scheduler consults the PACS algorithm, which in turn decides whether the subtask can be allowed to continue its execution. The system scheduler is assumed to share the task-specific information, usually called Process Control Block (PCB) or Task Control Block (TCB) of various applications with the PACS algorithm.

The pseudo-code of the algorithm is given in Figure 4. An illustration of the algorithm follows the pseudo-code. The parameters used in this algorithm are shown in Table 1. Note that a subtask belongs to an application, which in turn is a member of a class. So, while $A_{i}$ refers to the previous instance of abortion of a subtask belonging to an application $i, \delta_{i}$ denotes the loss constraint of class $i$ to which the application belongs.

If the algorithm can accommodate the requesting subtask, then the amount of 


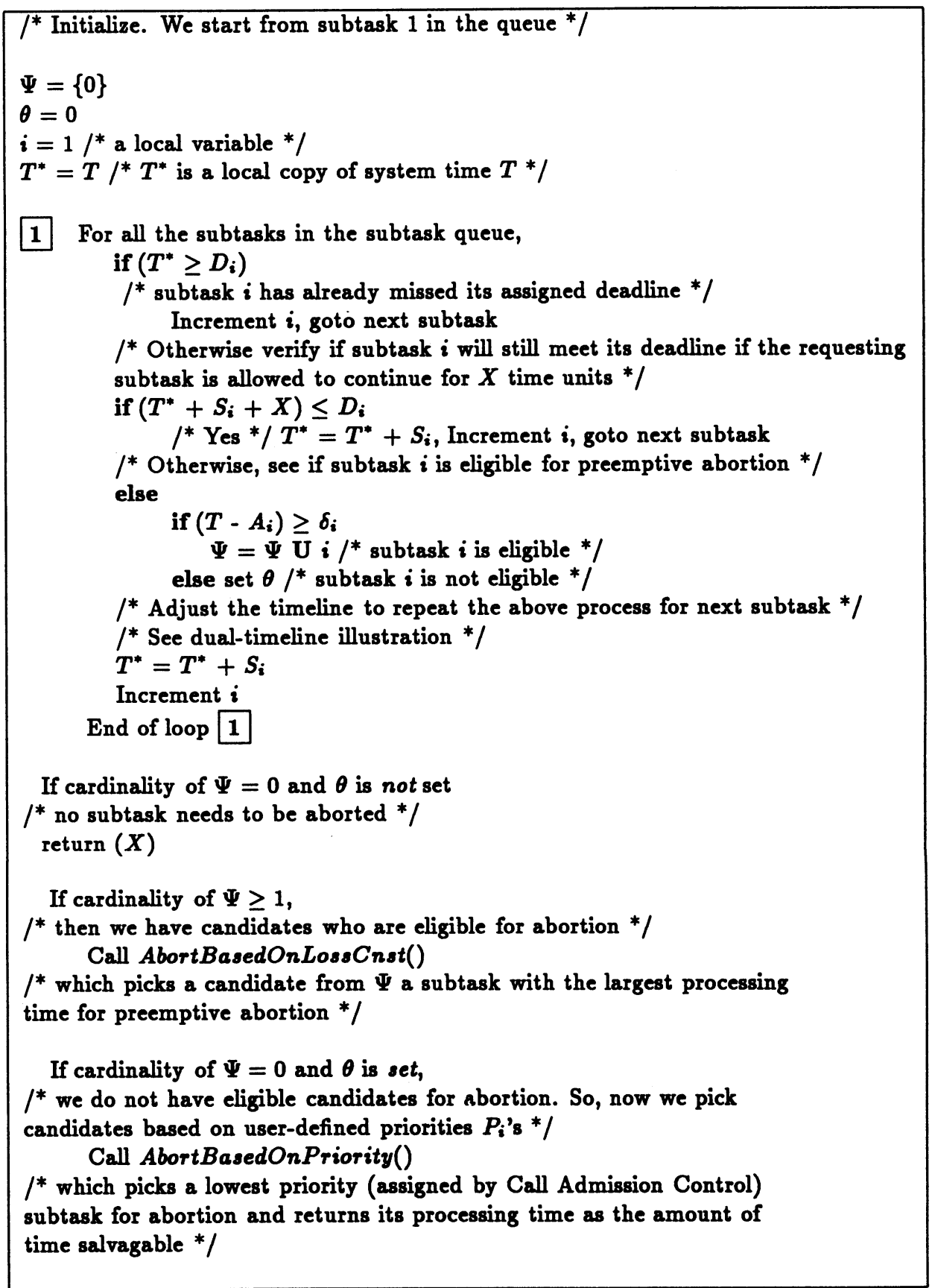

Figure 4: PACS Algorithm 


\begin{tabular}{|l|l|}
\hline$T$ & The current system time \\
\hline$X$ & Extra amount of service needed by the requesting subtask \\
\hline$S_{i}$ & The estimated service time of subtask $i$ \\
\hline$D_{i}$ & The assigned deadline of subtask $i$ \\
\hline$A_{i}$ & $\begin{array}{l}\text { Time of last abortion (preemptive or otherwise) } \\
\text { of a subtask belonging to application } i\end{array}$ \\
\hline$\delta_{i}$ & The nodal loss constraint of an application class $i$ \\
\hline$P_{i}$ & The priority of class $i$ \\
\hline$\Psi$ & Set of possible candidates for abortion \\
\hline$\theta$ & $\begin{array}{l}\text { A Flag, when set, implies preemptive abortion should } \\
\text { take place }\end{array}$ \\
\hline AbortBasedOnLossCnst() & Aborts a subtask based on loss constraint \\
\hline AbortBasedOnPriority () & Aborts a subtask based on the CAC-assigned priority \\
\hline
\end{tabular}

Table 1: PACS Algorithm Parameters

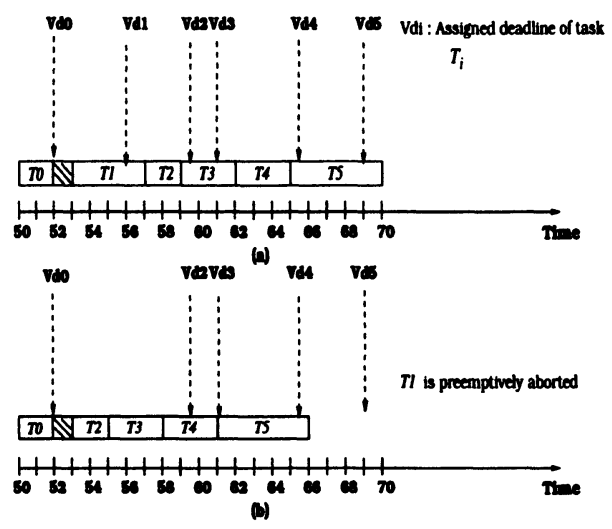

Figure 5: An illustration of the algorithm

salvaged time is returned to the scheduler, which allows the subtask to continue its service. Since the analysis is based on estimated execution times, it is possible that the requesting subtask may again undergo deadline expiration, in which case, the same process will be repeated. It is also possible that some other subtask which was involved in the analysis may itself undergo deadline violation for the same reason.

\subsection{Illustration of the PACS algorithm}

Example 2. Consider the subtasks shown in Figure 5. The subtask-specific information for each subtask is shown in Table 2. Note that such information is usually maintained as a part of the Process Control Block or Task Control Block data structure.

The subtask $T_{0}$ has undergone deadline expiration after executing for 2 units of time at time 52, and is requesting an additional processing time of 1 time unit (shown as a shaded box in Figure 5). At this time, the PACS algorithm is invoked to see if the request for additional time for subtask $T_{0}$ can be honored. The PACS 


\begin{tabular}{|c|c|c|c|c|c|}
\hline & $\begin{array}{c}\text { Estimated } \\
\text { Service Time }\end{array}$ & $\begin{array}{c}\text { Assigned } \\
\text { Deadline }\end{array}$ & $\begin{array}{c}\text { Loss } \\
\text { Constraint }\end{array}$ & $\begin{array}{c}\text { Previous Abortion } \\
\text { Instance }\end{array}$ & CAC-Priority \\
\hline$T_{0}$ & 2.0 & 52.0 & 25.0 & 30.0 & 2 \\
\hline$T_{1}$ & 4.0 & 56.0 & 30.0 & 15.0 & 3 \\
\hline$T_{2}$ & 2.0 & 59.5 & 30.0 & 5.0 & 3 \\
\hline$T_{3}$ & 3.0 & 61.0 & 30.0 & 0.0 & 3 \\
\hline$T_{4}$ & 3.0 & 65.5 & 20.0 & 20.0 & 1 \\
\hline$T_{5}$ & 5.0 & 69.0 & 25.0 & 35.0 & 2 \\
\hline
\end{tabular}

\section{Table 2: Subtask Parameters}

algorithm lays down the subtask profiles as shown in Figure 5(a), where the subtasks in the ready queue are scheduled from a new starting point determined by the amount of additonal request for subtask $T_{0}$. The subtasks $T_{1}, T_{3}$ and $T_{5}$ miss their assigned deadlines as a result of extending service to $T_{0}$. However, while checking the individual subtasks for their eligibility for preemptive abortion based on their nodal loss constraints and previous abortion instances, PACS finds $\Psi=\left\{T_{1}, T_{3}\right\}$. See Table 2. The algorithm then picks subtask $T_{1}$ for abortion, since its processing requirement is greater of the two subtasks in $\Psi$. Since the amount of time salvaged is greater than the amount requested, ( 4 time units vs 1 time unit), $T_{0}$ can continue its execution. The redrawn profiles are shown in Figure 5(b).

For more detailed illustration of the algorithm, please refer to [7].

\section{Performance Evaluation}

The system shown in Figure 1 was simulated. For each frame, there is an end-toend deadline and each corresponding application specifies a loss constraint. The loss constraint refers to the loss constraint for $B$ and $P$ frames, $I$ frames were not considered for preemptive abortion. The audio was assumed to have a separate channel and was not considered for preemptive abortion. So, the following discussion refers to video only. Also, we assume that the frame processing times at each node are estimated using exponential distribution ${ }^{1}$.

The frames from individual applications arrive at the multiplexer periodically, e.g., 30 frames every second. Even though the encoder outputs 30 compressed frames in a second, the output stream for each application from the encoder is not periodic due to the variance in compression times for individual frames. This results in occasional bursty arrivals at the application and the network schedulers. Assuming that the encoder outputs 30 frames in a second for each application, both the application and the network scheduler have to process 30 frames in a second per application. We achieve this by invoking the respective scheduler once every $33.33 \mathrm{~ms}$, which then runs all the subtasks in its queue. The queue itself is ordered according to the non-preemptive Earliest Deadline First discipline, i.e. the subtask with the most urgent deadline runs first during each invocation.

The performance measure of interest here is the cutoff load where the NLR

\footnotetext{
${ }^{1}$ Ofcourse the algorithm is not limited to any particular service time distribution.
} 
crosses the ANLR for each application class. See Figure 2. We do this with, and without, PACS. For both the cases, however, the nodal allocation of deadlines and loss constraints is performed according to the description in Section 3.1. The metric Relative Gain (RG) is defined as follows.

$$
R G=\frac{\text { cutoff }_{P A C S+}-\text { cutoff }_{P A C S-}}{\text { cutoffoPT }- \text { cutoff }_{P A C S-}}
$$

where cutoff PACS+ $_{\text {and }}$ cutoff ACS $_{-}$denote the cutoffs achieved with and without the PACS algorithm respectively. Cutoff $O P T$ is the upper bound on cutoff. Since cutoffoPT $\leq 1$, a lower bound of the relative gain is given by

$$
R G_{L}=\frac{\text { cutoff }_{P A C S+}-\text { cutoff }_{P A C S-}}{1-\text { cutoff }_{P A C S-}}
$$

A final note before we present the numerical results: we are not aware of similar work to compare our results with.

\subsection{Numerical Results}

We now focus on our first set of experiments. There are three classes of applications, each with a member application. The parameters values, the performance graphs, as well as the improvements made possible by the PACS algorithm for this set are presented in Figure $6{ }^{2}$. In Figure 6(a), the NLR is plotted versus the system load. It can be seen from this graph that Class 3 notices more losses than the other two due to its stricter loss constraint (even though at steady state all the three classes undergo approximately the same percentage of deadline misses). It can also be seen that the individual curves are spaced apart.

Figure 6(b) shows the performance of the three classes with the PACS scheme. The following observations can be made from this figure.

First, there is a considerable reduction in NLR for Class 2 and Class 3 applications. The overall improvement comes from two sources. First, where there is no abortion involved, simply additional processing time is borrowed from other subtasks without violating their assigned deadlines. This phenomenon alone appears to be the case until the load becomes moderately heavy (until about 0.6 ), and it continues along with preemptive abortion at loads beyond 0.6. Secondly, as the load increases (beyond 0.6), PACS aborts eligible candidates to make room for needy subtasks. Class 3 subtasks gain the most by aborting both Class 2 and Class 1 subtasks, since their loss constraints are less stringent. Class 2 subtasks compete with Class 3 subtasks to benefit from aborting Class 1 subtasks; they rarely get a chance to abort Class 3 subtasks. This explains why the improvement is higher for Class 3 subtasks compared to that for Class 2. There is a slight increase in NLR for Class 1 (represented by a negative $R G_{L}$ of $2.3 \%$ ) since a small percentage of its subtasks are aborted based on their priority. These priority-based abortions were counted as noticeable losses. However, recall that abortions that occur when the loss constraint allows PACS to do so are not noticeable losses.

The reason why preemptive abortion adds to considerable improvement in NLR (along with cycle stealing only) can be attributed as follows. PACS is a greedy algorithm which chooses an eligible subtask with maximum processing requirement when it has to perform abortion (based either on priority or loss constraint). And, it does this often during periods of burstiness or overloads during which normally many subtasks are lost resulting in

${ }^{2} \mathrm{~A}$ recent document by the Multimedia Communications Forum recommends using a terminal equipment (which includes video servers) delay of $200 \mathrm{~ms}$ and $150 \mathrm{~ms}$ for enhanced and premium quality multimedia communication respectively [11]. 

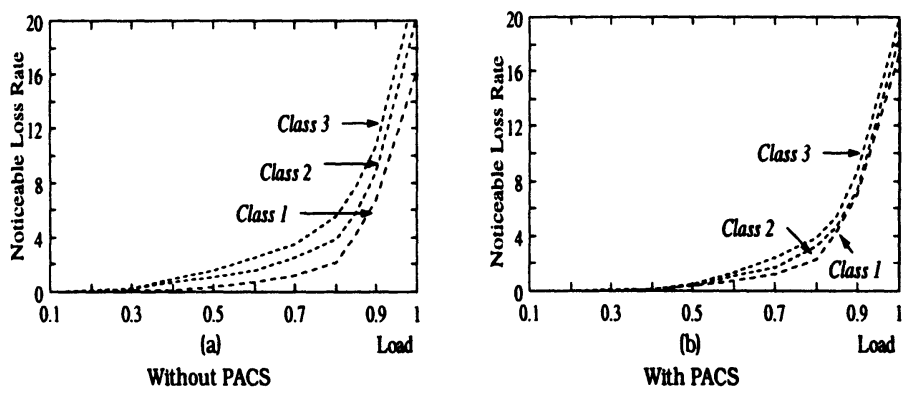

\begin{tabular}{|c|c|c|c|}
\hline & Class 1 & Class 2 & Class 3 \\
\hline Arrival Rate in frames/sec & 30 & 30 & 30 \\
\hline End-to-end Deadline & $200 \mathrm{~ms}$ & $200 \mathrm{~ms}$ & $200 \mathrm{~ms}$ \\
\hline Deadline Factor d & 1 & 1 & 1 \\
\hline Overall Loss Constraint & $600 \mathrm{~ms}$ & $1000 \mathrm{~ms}$ & $1500 \mathrm{~ms}$ \\
\hline Acceptable NLR & $5.5 \%$ & $3.3 \%$ & $2.2 \%$ \\
\hline Cutof $_{\text {pacs }}$ & 0.875 & 0.755 & 0.570 \\
\hline Cutof $_{\text {pacst }}$ & 0.872 & 0.800 & 0.675 \\
\hline Relative Gain $\boldsymbol{R G}_{L}$ & $-2.30 \%$ & $18.40 \%$ & $24.41 \%$ \\
\hline
\end{tabular}

Figure 6: Simulation Results: Case 1

higher NLR. By greedily salvaging time during such periods, PACS dampens the effect of burstiness, thus reducing clustered losses which would result in poor QoS.

Our next set of experiments include tighter end-to-end deadline and nodal deadline for all the three classes, as well as the addition of two more classes of applications. The details of these experiments and the performance of the PACS algorithm are available in [7].

\section{Conclusion}

In this paper, we have presented a resource management strategy for supporting multiple application classes with disparate QoS requirements. We have evaluated the performance of this scheme using a metric that suitably captures the loss tolerance abilities of soft realtime multimedia applications. Finally, we have shown through simulation experiments that the PACS approach indeed provides meaningful performance improvements in a practical application.

Our future work involves applying our loss model and PACS to bundled multimedia such as audio, video and graphics. We are implementing the PACS algorithm in a stored video server (for distance learning) for supporting rate and loss guarantees to individual user connections.

\section{Acknowledgement}

This work was supported in part by the National Science Foundation under grant CCR-911 9922 


\section{References}

[1] R. L. Cruz. "A Calculus for Network Delay, Part II: Network Analysis" IEEE Transactions on Information Theory, Vol 37, No. 1, Jan 1991.

[2] C. Douglass Locke. "Software Architecture for Hard Real-Time Applications: Cyclic Executives vs Fixed Priority Executives" The Journal of Real-Time Systems, vol4(1), pp 37-53, March 1992.

[3] D. Ferrari and D. Verma. "A Scheme for Real-time Channel Establishment in WideArea Networks". IEEE Journal on Selected Areas in Communication. Vol. 8, No. 3, pp 368-379, April 1990.

[4] R. D. Gaglianello and G. L. Cash. "Montage: Continuous Presence Teleconferencing Utilizing Compressed Domain Video Bridging". Multimedia Communications Research lab report, AT\&T Bell Laboratories, Holmdel, NJ 07733. 1994-95.

[5] B. Kao and H. Garcia-Molina, "Deadline Assignment in a Distributed Soft Real-Time System" Proceedings of the 19th International Conference on Distributed Computing Systems, pp 428-437, 1993.

[6] R. Koodli and C. M. Krishna. "Supporting End-to-End Deadlines for Soft Real-time Multimedia Applications". In IEEE International Performance, Computing and Communications Conference, February 5-7, 1997.

[7] R. Koodli and C. M. Krishna "Supporting Multiple-tier QoS in a Video Bridging Application" TR-CSE-97-2, ECE department, University of Massachusetts, Amherst, MA 01003. ftp://aurelius.ecs.umass.edu/pub/koodli/qos.ps.Z

[8] J. Kurose. "On Computing Per-Session Performance Bounds in High-Speed Multi-Hop Computer Networks", In ACM SIGMETRICS'92, pp 128-139, 1992.

[9] R. Nagarajan, J. Kurose and D. Towsley. "Nodal Allocation of End-to-End QoS in High-Speed Networks." IFIP, January 1993.

[10] C. L. Liu and J. W. Layland. "Scheduling Algorithms for Multiprogramming in a Hard Real-Time Environment" Journal of the Association for Computing Machinery, vol 20(1), pp 46-61, 1973.

[11] "Multimedia Communications Quality of Service, Part 2: Multimedia Desktop Collaboration Requirements". Final document, Multimedia Communications Forum Inc. Suite 201-931 Brunette Avenue Coquitlam, British Columbia, Canada V3K 6T5.

[12] "REACT Real-time Programmers Guide" Silicon Graphics Technical Publications, Document number 007-2499-002.

[13] H. Schulgrinne. "Reducing and Charcterizing Packet Loss for High-Speed Computer Networks with Real-Time Services" PhD dissertation, University of Massachusetts, Amherst, Computer Science Technical Report 99-54, 1993.

[14] A. Yan, A. Ganz, and C. M. Krishna, "A Distributed Adaptive Protocol Providing Real-Time Services on WDM Based LANs", IEEE Journal of Lightwave Technology Vol 45, pp 753-756, June 1996.

\section{Biography}

Rajeev Koodli is a $\mathrm{PhD}$ candidate in the ECE department at the University of Massachusetts, Amherst. His research interests include scheduling support for real-time multimedia, QoS in video applications and distributed systems.

C. M. Krishna is a professor in the ECE department at the University of Massachusetts, Amherst. His research interests are real-time systems, fault-tolerance and real-time recovery, distributed systems and high-speed networks. 